\title{
STABLE MEASURE OF A SMALL BALL
}

\author{
M. LEWANDOWSKI, M. RYZNAR, AND T. ŻAK
}

(Communicated by Lawrence F. Gray)

\begin{abstract}
Let $\mu$ be a symmetric $p$-stable measure on a Banach space $(E$, $\|\cdot\|)$. We prove that $\mu\{\|x\|<t\} \leq K t$, where the constant $K$ is independent of all properties of $\mu$ except for the measure of the unit ball $\mu\{\|x\|<1\}$.
\end{abstract}

\section{INTRODUCTION AND PRELIMINARY FACTS}

Let $\mu$ be a symmetric Gaussian measure on a separable Banach space $(E$, $\|\cdot\|)$. In the paper of Szarek [7] there is the following bound for the distribution function of the norm

$$
\mu\left(B_{t}\right) \leq K t, \quad t>0,
$$

where $B_{t}$ is the ball with radius $t$ and $K$ is a constant depending only on $\mu\left(B_{1}\right)$. This inequality was used to obtain some results in the theory of computational complexity [7].

A similar result in the Hilbert space case was proved by Sawa [6]

$$
\mu\left(B_{t}\right) \leq \Phi(t), \quad 0<t \leq 0,2
$$

provided $\int\|x\|^{2} d \mu(x)=1$ and $\Phi(t)=\sqrt{\frac{2}{\pi}} \int_{0}^{t} \exp \left(-x^{2} / 2\right) d x$.

The purpose of this note is both to extend (1) to the case of symmetric $p$ stable measures, $0<p \leq 2$, and to give another proof for (1). Our method consists in using a series representation of stable random vectors obtained in [4].

Let us recall that a symmetric measure $\mu$ is called $p$-stable, $0<p \leq 2$, iff for every $t, s>0$ we have

$$
t X+s Y \stackrel{\mathrm{d}}{=}\left(s^{p}+t^{p}\right)^{1 / p} X
$$

where $X, Y$ are i.i.d. random vectors with the distribution $\mu$. It is well known (see, e.g., [5]) that there exists a finite measure $\sigma$ on $S_{1}=\{x:\|x\|=1\}$ such that the characteristic functional of $\mu$ has the form

$$
\hat{\mu}\left(x^{*}\right)=\exp \left(-\int\left|x^{*}(x)\right|^{p} \sigma(d x)\right), \quad x^{*} \in E^{*} .
$$

Received by the editors November 26, 1990 .

1980 Mathematics Subject Classification (1985 Revision). Primary 60B11, 60 E07.

Key words and phrases. Stable measure, Gaussian measure, distribution of the norm.

This paper was presented by the second author on June 1, 1990 during Banach Center Probability Workshop in Warsaw, Poland. 
The measure $\sigma$ is called the spectral measure of $\mu$.

In the following lemmas we recall a series representation of $p$-stable random vectors in $E$. Let $\left(\alpha_{i}\right)_{i=1}^{\infty}$ and $\left(z_{i}\right)_{i=1}^{\infty}$ be two sequences of i.i.d. random variables such that $P\left(\alpha_{1}>t\right)=e^{-t}, E\left|z_{1}\right|^{p}=1$, and $z_{1}$ is symmetric. We assume that $\left(\alpha_{i}\right)$ and $\left(z_{i}\right)$ are independent. Next, we denote $\Gamma_{n}=\alpha_{1}+\alpha_{2}+\cdots+\alpha_{n}$ and

$$
c_{p}=\left(\int_{0}^{\infty} v^{-p} \sin v d v\right)^{-1 / p}=\left(\frac{1-p}{\Gamma(2-p) \cos \pi \frac{p}{2}}\right)^{1 / p} .
$$

Lemma 1 [4]. (a) For $0<p<2$ the series $c_{p} \sum_{i=1}^{\infty} \Gamma_{i}^{-1 / p} z_{i}$ is convergent a.s. and the characteristic function of the limit is $\exp \left(-|t|^{p}\right)$.

(b) For $0<p<1$ the series $\sum_{i=1}^{\infty} \Gamma_{i}^{-1 / p}$ is convergent a.s. and has $p$-stable distribution with the characteristic function

$$
\exp \left(-c_{p}|t|^{p}\left(1-i(\operatorname{sgn} t) \operatorname{tg} \pi \frac{p}{2}\right)\right) .
$$

Lemma 2 [4]. Let $\mu$ be a symmetric p-stable measure, $0<p<2$, with the spectral measure $\sigma$. Let $\left(V_{i}\right)_{i=1}^{\infty}$ be a sequence of i.i.d. random vectors with the distribution $\sigma / \sigma\left(S_{1}\right)$, independent of $\left(\alpha_{i}\right)$ and $\left(z_{i}\right)$. Then the series

$$
c_{p}\left[\sigma\left(S_{1}\right)\right]^{1 / p} \sum_{i=1}^{\infty} \Gamma_{i}^{-1 / p} V_{i} z_{i}
$$

is convergent a.s. and has the distribution $\mu$.

\section{THE MAIN RESULT}

Theorem. Suppose that $\mu$ is a symmetric p-stable measure, $0<p \leq 2$, such that $\int\|x\|^{r} \mu(d \mu)=1$ for some $r \in(0, p)$. There exists a constant $K(p, r)$ depending only on $p$ and $r$ so that

$$
\mu\left(B_{t}\right) \leq K(p, r) t, \quad t>0 .
$$

The proof consists of two steps. In the first one we find the appropriate estimate for $\mu\left(B_{t}\right)$ provided $\sigma\left(S_{1}\right)=1$.

Step 1. We use Lemma 2 assuming that $\left(z_{i}\right)_{i=1}^{\infty}$ is a Gaussian sequence and $\sigma\left(S_{1}\right)=1$. Suppose that $\left(z_{i}\right)$ is defined on a probability space $\left(\Omega_{1}, P_{1}\right)$ and $\left(\alpha_{i}\right),\left(V_{i}\right)$ are defined on $\left(\Omega_{2}, P_{2}\right)$. When we fix $\left(\alpha_{i}\right)$ and $\left(V_{i}\right)$ then the random vector $c_{p} \sum_{i=1}^{\infty} \Gamma_{i}^{-1 / p} V_{i} z_{i}\left(\omega_{1}\right)$ is Gaussian. By Anderson's inequality [1] we have:

$$
\begin{aligned}
P_{1}\left(\left\|c_{p} \sum_{i=1}^{\infty} \Gamma^{-1 / p} V_{i} z_{i}\left(\omega_{1}\right)\right\| \leq t\right) \\
\quad=P_{1}\left(\left\|c_{p} \Gamma_{1}^{-1 / p} V_{1} z_{1}\left(\omega_{1}\right)+\sum_{i=2}^{\infty} c_{p} \Gamma_{i}^{-1 / p} V_{i} z_{i}\left(\omega_{1}\right)\right\| \leq t\right) \\
\quad \leq P_{1}\left(\left\|c_{p} \Gamma_{1}^{-1 / p} V_{1} z_{1}\left(\omega_{1}\right)\right\| \leq t\right) \\
\quad=P_{1}\left(\left|z_{1}\right| \leq c_{p}^{-1} \Gamma_{1}^{1 / p} t\right) \quad P_{2}-\text { a.s. }
\end{aligned}
$$

since $\left\|V_{1}\right\|=1 \quad P_{2}$-a.s. Therefore, by Fubini theorem

$$
\mu\left(B_{t}\right) \leq P_{1} \times P_{2}\left(\left|z_{1}\left(\omega_{1}\right)\right| \leq \Gamma_{1}^{1 / p}\left(\omega_{2}\right) c_{p}^{-1} t\right)=\Psi_{p}(t),
$$


where $\Psi_{p}$ is the distribution function of $\left|z_{1}\right| \Gamma_{1}^{-1 / p} c_{p}$.

Step 2. In this step we prove the theorem for $p$-stable measure $\mu, 0<p \leq 2$, provided $\int\|x\|^{r} d \mu(x)=1, r<p$. Let $\left(X_{i}\right)_{i=1}^{\infty}$ be a sequence of i.i.d. random vectors independent of $\left(\alpha_{i}\right)$ and with the distribution of $X_{1}$ equal to $\mu$. Put

$$
Y=c_{r} \sum_{i=1}^{\infty} \Gamma_{i}^{-1 / r} X_{i}
$$

By Lemma 1 the characteristic functional of $Y$ is equal to

$$
\begin{aligned}
\exp -\int\left|x^{*}(x)\right|^{r} \mu(d x) & =\exp -\int\left|x^{*}\left(\frac{x}{\|x\|}\right)\right|^{r}\|x\|^{r} \mu(d x) \\
= & \exp -\int\left|x^{*}(x)\right|^{r} \sigma_{Y}(d x), \\
& \text { where } \sigma_{Y}\left(S_{1}\right)=\int\|x\|^{r} \mu(d x)=1 .
\end{aligned}
$$

Hence $Y$ is an $r$-stable symmetric random vector with the spectral measure $\sigma_{Y}$ so that by Step $1, P(\|Y\|<t) \leq \Psi_{r}(t)$. On the other hand by the property (2) and the independence of $\left(\Gamma_{i}\right)$ and $\left(X_{i}\right)$ we obtain

$$
Y \stackrel{\mathrm{d}}{=} c_{r}\left(\sum_{i=1}^{\infty} \Gamma_{i}^{-p / r}\right)^{1 / p} X_{1}
$$

The series $\sum_{i=1}^{\infty} \Gamma_{i}^{-p / r}=\eta\left(\frac{r}{p}\right)$ is convergent a.s. by Lemma 1 and $\eta\left(\frac{r}{p}\right)$ is independent of $X_{1}$. Therefore, for every $a>0$ :

Hence

$$
\begin{aligned}
\Psi_{r}(a t) & \geq P(\|Y\| \leq a t)=P\left(c_{r}\left[\eta\left(\frac{r}{p}\right)\right]^{1 / p}\left\|X_{1}\right\| \leq a t\right) \\
& \geq P\left(c_{r} \eta^{1 / p} \leq a,\left\|X_{1}\right\| \leq t\right)=P\left(\eta \leq\left(\frac{a}{c_{r}}\right)^{p}\right) \cdot P\left(\left\|X_{1}\right\| \leq t\right) .
\end{aligned}
$$

$$
\mu\left(B_{t}\right) \leq \frac{\Psi_{r}(a t)}{P\left(\eta \leq\left(\frac{a}{c_{r}}\right)^{p}\right)} .
$$

Simple calculations give $\sup _{t>0} \Psi_{r}^{\prime}(t)=C<\infty$. Putting

$$
K(p, r)=\sup _{t>0} \Psi_{r}^{\prime}(t) \cdot \inf _{a>0} a\left[P\left(\eta \leq\left(\frac{a}{c_{r}}\right)^{p}\right)\right]^{-1}
$$

we get the desired conclusion.

For arbitrary values of $\int\|x\|^{r} \mu(d x)$ we obtain, by the Čebyshev inequality,

$$
\begin{aligned}
\mu\left(B_{t}\right) & \leq K(p, r)\left[\int\|x\|^{r} \mu(d x)\right]^{-1 / r} \cdot t \\
& \leq K(p, r)\left[1-\mu\left(B_{1}\right)\right]^{-1 / r} \cdot t .
\end{aligned}
$$

This inequality is a version of (1) for stable case $p<2$ and for $p=2$ it is precisely (1).

\section{Estimation of CONSTANTS $K(p, r)$}

First we estimate the value of $\sup _{t>0} \Psi_{r}^{\prime}(t)$. Recall $\Psi_{r}(t)=P\left(\left|z_{1}\right| \Gamma_{1}^{-1 / r} c_{r} \leq t\right)$, $z_{1}$ has the distribution $N\left(0, \sigma_{r}\right)$, where $\sigma_{r}=\pi^{1 / 2 r}\left(\sqrt{2} \Gamma^{1 / r}\left(\frac{r+1}{2}\right)\right)^{-1}, c_{r}=$ 
$\left((1-r) / \Gamma(2-r) \cos \pi \frac{r}{2}\right)^{1 / r}$, and $c_{1}=\frac{2}{\pi}$.

Now

$$
\begin{aligned}
\Psi_{r}^{\prime}(t) & =\int_{0}^{\infty} \frac{d}{d t} P\left(\left|z_{1}\right| \leq \frac{t}{c_{r}} x^{1 / r}\right) e^{-x} d x \\
& =\int_{0}^{\infty} \frac{\sqrt{2}}{\sqrt{\pi} \sigma_{r}} \exp \left(-\frac{x^{2 / r} t^{2}}{2 c_{r}^{2} \sigma_{r}^{2}}\right) \frac{x^{1 / r}}{c_{r}} e^{-x} d x \\
& \leq \sqrt{\frac{2}{\pi}} \frac{\Gamma(1+1 / r)}{c_{r} \sigma_{r}}=A_{r} .
\end{aligned}
$$

We will also need an evaluation of the distribution function of $\eta\left(\frac{1}{2}\right)=$ $\sum_{i=1}^{\infty} \Gamma_{i}^{-2}$ which, by Lemma $1(\mathrm{~b})$, has $\frac{1}{2}$-stable distribution on $R^{+}$with the density $f(x)=1 /\left(2 x^{3 / 2}\right) \exp (-\pi /(4 x))$ for $x>0$. It is easy to notice that $P\left(\eta\left(\frac{1}{2}\right)<t\right)=1-\Phi\left(\sqrt{\frac{\pi}{2 t}}\right)$.

(a) $p=2, r=1$. This is the Gaussian case. Assume that $\int\|x\| \mu(d x)=1$. By (5),

$$
\begin{aligned}
\mu\left(B_{t}\right) & \leq A_{1} \cdot \inf _{a>0} a\left[P\left(\eta\left(\frac{1}{2}\right) \leq\left(\frac{a}{c_{1}}\right)^{2}\right)\right]^{-1} t \\
& =A_{1} \sqrt{\frac{2}{\pi}} c_{1}\left[\sup _{r>0} r(1-\Phi(r))\right]^{-1} t \leq 2,35 t,
\end{aligned}
$$

because $A_{1}=1, c_{1}=\frac{2}{\pi}$, and the above supremum is attained in a neighborhood of $r=0,75$.

(b) For arbitrary $p \in(0,2)$ we take $r=\frac{p}{2}$ (because in this case we know the density of $\left.\eta\left(\frac{r}{p}\right)\right)$. In this case

$$
\begin{aligned}
K\left(p, \frac{p}{2}\right) & =A_{p / 2} \inf _{a>0} a\left[P\left(\eta\left(\frac{1}{2}\right) \leq\left(\frac{a}{c_{p / 2}}\right)^{p}\right)\right]^{-1} \\
& =\frac{1}{2^{1 / p} \sqrt{\pi}} \Gamma^{2 / p}(p / 4+1 / 2) \Gamma(1+2 / p) \inf _{r>0}\left(r^{2 / p}(1-\Phi(r))\right)^{-1} .
\end{aligned}
$$

Observe $K\left(p, \frac{p}{2}\right)$ tends to infinity very rapidly as $p \rightarrow 0$. But, when $p \geq$ $\varepsilon>0$ we can find some upper bound for it. For example, if $1 \leq p<2$ then the properties of the function $\Gamma(x)$ give the estimate $\mu\left(B_{t}\right) \leq 2 \sqrt{2 \pi} /(1-\Phi(1)) \cdot t \leq$ $15,8 t$, when we take $r=1$ in (6) for simplicity.

(c) If $0<p<1$ we can give an estimate better than (6).

It is well known that in Banach spaces of stable type $p$ there holds an inequality between the $r$ th moment of a $p$-stable measure and the total mass of its spectral measure, and every Banach space is of stable type $p, p<1$ (see, e.g., [5]). Namely, as it was shown by Pisier [2, Lemma 5.4]: if $X$ is a $p$-stable random vector, $0<p<1,0<r<p$, and $\sigma$ is its spectral measure then

$$
\left[E\|X\|^{r}\right]^{1 / r} \leq \frac{c_{p}(r) c_{1}(p)}{c_{1}(r)}\left[\sigma\left(S_{1}\right)\right]^{1 / p},
$$

where $c_{p}^{r}(r)=2^{r-1} \Gamma(1-r / p)\left(r \int_{0}^{\infty} u^{-r-1} \sin ^{2} u d u\right)^{-1}$ denotes the $r$ th moment of the standard symmetric $p$-stable random variable on $R$ (for the value of 
$c_{p}(r)$ compare [3]). But

$$
\begin{aligned}
r \int_{0}^{\infty} u^{-r-1} \sin ^{2} u d u & =2^{r-1} \int_{0}^{\infty} u^{-r} \sin u d u=2^{r-1} c_{r}^{-r} \\
& =2^{r-1} \Gamma(2-r) \cos \frac{\pi r}{2} \cdot[(1-r)]^{-1}
\end{aligned}
$$

Finally

$$
c_{p}(r)=\left[\frac{\Gamma(1-r / p)(1-r)}{\Gamma(2-r) \cos \frac{\pi r}{2}}\right]^{1 / r}
$$

and, by (7),

$$
\begin{aligned}
\left(E\|X\|^{r}\right)^{1 / r} & \leq\left[\frac{\Gamma(1-r / p)}{\Gamma(1-r)}\right]^{1 / r}\left[\frac{\Gamma(1-p)(1-p)}{\Gamma(2-p) \cos \frac{\pi r}{2}}\right]^{1 / p} \cdot\left[\sigma\left(S_{1}\right)\right]^{1 / p} \\
& =B_{p}(r)\left[\sigma\left(S_{1}\right)\right]^{1 / p}
\end{aligned}
$$

We use only Step 1. If $\mu$ is symmetric $p$-stable with the spectral measure $\sigma$ then by (4) and (7) and the definitions of $A_{p}$ and $B_{p}(r)$ :

$$
\begin{aligned}
\mu\left(B_{t}\right) & \leq \frac{\Psi_{p}(t)}{\left[\sigma\left(S_{1}\right)\right]^{1 / p}} \\
& \leq \frac{\Psi_{p}(t) B_{p}(r)}{\left[\int\|x\|^{r} \mu(d x)\right]^{1 / r}} \leq \frac{A_{p} B_{p}(r)}{\left[\int\|x\|^{r} \mu(d x)\right]^{1 / r}} \cdot t .
\end{aligned}
$$

Remarks. (1) If $p$ is a concrete given number we can estimate more carefully in (6) and get better constant.

(2) For symmetric $p$-stable random variable on $R$ with characteristic function $\exp \left(-|t|^{p}\right)$ we have $P(|X|<t) \leq \pi^{-1} \Gamma(1+1 / p) t$ because $\Gamma(1+1 / p) / \pi$ is precisely the value of the density at zero, hence the constant $A_{p} B_{p}(r)$ must tend to infinity at least like $\Gamma(1+1 / p)$ when $p \rightarrow 0$.

\section{ACKNOWLEDGMENT}

This problem arose during the Seminar on Probability in Banach Center (Warsaw, May 1990). We would like to thank Professor S. Kwapień for stimulating conversations and for showing preprint [7] to us.

Added in proof. When the first draft of this paper was circulating, the second author had a talk on this subject during the Banach Center Probability Workshop (June 1990). After his talk Professor X. Fernique kindly informed us that for the Gaussian case $(p=2)$ he knew another two proofs. One is explicitly contained in his paper Les vecteurs aléatoires gaussiens et leurs espaces autoreproduisants, Technical Report 34, Ser. Lab. Res. Statist. and Probab., University of Ottawa, 1985.

For the second, one can use an inequality of Kanter (Probability inequalities for convex sets and multidimensional concentration functions, J. Multivariate Anal. 6 (1976), 222-236, inequality 4.1): for $\left(X_{i}\right)_{i=1}^{n}$ independent and symmetric

$$
P\left(\left\|\sum_{i=1}^{n} X_{i}\right\|<t\right) \leq\left(\frac{3}{2}\right)\left(1+\sum_{i=1}^{n} P\left(\left\|X_{i}\right\|>t\right)\right)^{-1 / 2} .
$$


Taking $X_{1} \stackrel{\mathrm{d}}{=} X_{2} \stackrel{\mathrm{d}}{=} \ldots \stackrel{\mathrm{d}}{=} X / \sqrt{n}$ and $n=\left[\frac{1}{t^{2}}\right]$ we get the desired conclusion.

Fernique's proof is based on the rotational invariance of the product of Gaussian measures; hence it does not immediately apply for $p<2$. The second method gives the estimate of order $t^{p / 2}$, which is worse than $t$ for $t \rightarrow 0$, if $p<2$.

\section{REFERENCES}

1. T. Anderson, The integral of symmetric unimodal functions over a symmetric convex set and some probability inequalities, Proc. Amer. Math. Soc. 6 (1955), 170-176.

2. S. A. Chobanian, V. I. Tarieladze, and N. N. Vakhania, Probability distributions on Banach spaces, Reidel, Dordrecht, 1987.

3. C. D. Hardin, Jr., Skewed stable variables and processes, Technical Report 79, Center for Stochastic Proc., Univ. of North Carolina, Chapel Hill, 1984.

4. R. Le Page, M. Woodroofe, and J. Zinn, Convergence to a stable distribution via order statistics, Ann. Probab. 9 (1981), 624-632.

5. W. Linde, Infinitely divisible and stable measures on Banach spaces, Wiley, New York, 1986.

6. J. Sawa, private communication, 1990.

7. S. Szarek, Condition numbers of random matrices, preprint, 1989.

Technical University, Department of Mathematics, Wybrzeże Wyspianskiego 27, WroClaw 50 370, Poland 